\title{
Análisis crítico del entorno, elección de alternativas y clarificación de valores
}

\section{Isabel Carrillo}

\section{$\mathcal{D} \mathcal{M a}$}

En el presente artículo se relacionan el conjunto de estrategias conocidas bajo el epígrafe de «clarificación de valores» con el desarrollo de la personalidad moral. Se especifican las fases a tener en cuenta en su puesta en práctica, ejemplificándose en actividades dirigidas en especial a los alumnos del tercer ciclo de la Educación Primaria, ofreciéndose prototipos de actividades para otros tramos educativos.

\section{ASPECTOS TEORICOS}

\section{Introducción}

La técnica de clarificación de valores engloba un conjunto de métodos de trabajo cuya finalidad es ayudar a los niños y niñas, y jóvenes, a realizar un proceso de reflexión orientado a tomar conciencia de las propias valoraciones, opiniones y sentimientos.

El origen de esta técnica se sitúa en las aportaciones de L. Raths y sus colaboradores que, en 1966, publicaron la obra «Values and Teaching» ${ }^{1}$. Estas aportaciones teóricas surgen en un contexto histórico caracterizado por la gran diversidad de valores, hecho que genera, en las jóvenes generaciones, conflictors y cierta confusión sobre lo que es correcto, justo, adecuado,... Ante esta desorientación, la clarificación de valores se presenta como un medio que permite a la persona integrar el pensamiento, la afectividad y la acción.

Desde el ámbito de la educación moral, y de acuerdo a los modelos basados en la construcción racional y autónoma de valores, se incorpora esta técnica con la finalidad de promover, entre los alumnos/as, los procesos de autoconocimiento $\mathrm{y}$ expresión de valores ${ }^{2}$. 


\section{Clarificación de valores y personalidad moral}

Como hemos indicado, la clarificación de valores contribuye a que los alumnos/as realicen un proceso de reflexión que les permitirá tomar conciencia y responsabilidad respecto a sus pensamientos, sus valoreš, y sus emociones. Este proceso ayuda, por una parte, a adquirir más confianza y seguridad en sí mismo y, por otra, a enfrentarse constructivamente a las situaciones conflictivas, posibilitando una participación más consciente en una sociedad democrática.

Así, mediante un método explícito y estructurado, se realiza un análisis introspectivo que permite reflexionar acerca de la personalidad, los comportamientos, las actitudes, los valores... Este análisis, que ha de basarse en el uso de la razón y el diálogo con uno mismo, es necesario para que los niños, niñas y jóvenes, vayan construyendo su personalidad moral. No se trata por tanto de una estrategia orientada hacia la imposición de valores, sino más bien al contrario, desde nuestra perspectiva la clarificación de valores ha de ser el medio que permita a la persona, de forma racional y autónoma, descubrirse a sí misma y, al mismo tiempo, iniciar procesos de cambio - personal y colectivos-. En este sentido, el autoconocimiento es un requisito para establecer pautas autorreguladoras y de autocontrol de la propia conducta, es decir, de la propia acción.

En síntesis, la acción pedagógica ha de fomentar el desarrollo de la personalidad moral de los alumnos/as y, para ello, no ha de olvidar el desarrollo de procedimientos que permitan un progresivo autoconocimiento, en el sentido de descubrirse a sí mismo y, con ello, iniciar un proceso de construcción y valoración positiva del yo, integrando la experiencia biográfica y la proyección hacia el futuro. El fin último es facilitar tanto el proceso continuo de clarificación personal, como el uso de estrategias para conseguirlo.

\section{El proceso de valoración}

La clarificación de valores supone un proceso que permita a la persona analizar, de manera crítica y creativa, su entorno, a la vez que le ayuda a escoger, de forma autónoma y racional, sus principios de valor. En este sentido la reflexión -interna y externa - permite conocer y clarificar lo que cada uno valora y aprecia, así como las propias opiniones y sentimientos, ante los diferentes conflictos de valor que plantea la realidad.

En este proceso de reflexión y de valoración personal se han de seguir tres fases: 1) Selección. Proceso cognitivo que requiere libertad para decidir lo que. cada uno valora y aprecia. Esta decisión se toma después de considerar las diferentes alternativas y analizar las consecuencias de cada una de ellas; 2) Apreciación, donde interviene la afectividad en el sentido de que la persona ha de apreciar y disfrutar de su elección, estando dispuesta a defenderla públicamente; 3) Actuación, referida a la conducta, es decir, la persona ha de actuar de acuerdo a su elección, ya que lo que valoramos y apreciamos ha de constituirse en guía de nuestra vida.

Este proceso de valoración, que permite tomar conciencia de lo que valoramos y apreciamos, lo que pensamos y sentimos, respecto a temas conflictivos, puede realizarse a través de diversos métodos, si bien vamos a centrarnos en las frases inacabadas y preguntas clarificadoras ${ }^{3}$. Este método, de carácter individual, consiste en presentar una serie de frases inacabadas, y/o preguntas, que obligan al alumno/a a definirse, es decir, se le ofrece la oportunidad de pensar 
sobre sus creencias, sus opiniones, sus preferencias, sus valores, sus actitudes..., en relación a un tema de carácter ético.

\section{ASPECTOS PRACTICOS: FRASES INACABADAS Y PREGUNTAS CLARIFICADORAS}

Como ya hemos indicado, si bien son varios los métodos que permiten a la persona realizar un proceso de valoración, vamos a centrarnos en las frases inacabadas y preguntas clarificadoras. Estos ejercicios se han de realizar a propósito de temas que puedan resultar controvertidos e interesantes para los alumnos/as, a la vez que han de ser moralmente relevantes. Es importante que, previamente a la realización de estos ejercicos, se haya abordado el tema desde otras perspectivas, sobre todo aquellos que aportan información y permiten una comprensión crítica del mismo. A continuación presentamos una actividad, a modo de ejemplo, donde se realiza este trabajo previo a la clarificación de valores.

\section{Actividad}

«La Constitución» (Ambito temático: Elementos conceptuales y filosóficos. Curso: $6 .^{\circ}$ de EGB).

Tema/Objetivo: En este actividad se aborda el tema de la Constitución Española de 1978, su significado así como los aspectos que regula —organización y vida en común de las personas-. Consideramos que estos contenidos pueden someterse al proceso de valoración, ya que son diversas las situaciones sociales en las que se plantean conflictos de valor en relación a los principios declarados en esta ley fundamental. Por ello es necesario que el alumno/a conozca la Constitución y tome postura respecto a su función de guía de la convivencia en una sociedad democrática.

Cómo aplicar la actividad: La actividad se divide en dos partes. En la primera se aporta información al alumno/a y se realiza un ejercicio de comprensión crítica ${ }^{4}$. En la segunda parte se presenta un ejercicio de clarificación de valores - frases inacabadas-.

La lectura del texto sirve como elemento introductorio del tema, a nivel conceptual. Mediante la contestación de las preguntas, que se realizan a continuación, se favorece la comprensión crítica del tema. Esta comprensión se consigue también entablando un diálogo en torno a los conceptos que se presentan en el texto. A partir de aquí se puede iniciar la realización de la segunda parte de la actividad, es decir, el proceso de valoración, mediante el ejercicio de frases inacabadas a propósito de los conceptos/valores planteados en el texto. Este ejercicio, de carácter individual, permite al alumno/a realizar un proceso de reflexión personal dirigido a definirse a sí mismo y, mediante sus respuestas, manifestar sus opiniones y su postura.

\section{Actividad}

a) Información/Comprensión Crítica:

Lee la siguiente historia, en ella se explica como nace una Constitución y cuál es su finalidad.

Hace muchos años, tantos que ya no recuerdo cuando fue, en una pequeña isla del Pacífico vivía un hombre que llamaremos Quim. No sabemos como llegó a esta 
isla, pero debía hacer muchos años pues Quim tenía una barbas tan largas que casi le llegaban a los pies. Este hombre se había construido una cabaña con la madera de los árboles; vivía de algunas plantas que recogía en el bosque y, también, de la caza y la pesca.

Un día Quim escuchó un terrible ruido. Era el ruido de una pequeña avioneta que había caído en picado en medio del bosque. Aunque la avioneta quedó destrozada, el hombre y la mujer que iban en ella se salvaron, pero claro, ahora ya no tenían ningún medio para volver a su país $y$, por tanto, se tuvieron que quedar a vivir para siempre en la isla.

A Quim no le gustó demasiado la presencia de estos dos intrusos en su territorio $y$, por esto, su primer pensamiento fue echarlos fuera por cualquier medio. Pero inmediatamente se dio cuenta de que aquellos intrusos eran dos personas sanas, jóvenes y fuertes y que, si intentaba luchar con ellos, no había duda de que le ganarian. Así, antes de que se les ocurriera utilizar su fuerza para quedarse en la isla, el viejo Quim les ofreció una parte de sus territorios de caza y pesca para que pudieran vivir.

Un día de principios de abril comenzó a llover. Los días pasaban y la lluvia no paraba. El viento soplaba de miedo y el mar parecía que chillaba espantado de tanta agua. Una noche el mar estaba tan enfadado que lanzó a la playa un barco destrozado por la gran tempestad que había soportado. De su interior salieron cincuenta personas, grandes y pequeños, hombres y mujeres. Al día siguiente vieron como había quedado el barco, estaba tan lleno de agujeros que era imposible ponerlo a navegar de nuevo. No les quedó otra solución que quedarse a vivir en la isla.

La llegada de todas estas personas lo complicó todo. La isla era muy pequeña y no podían dividir más los territorios de caza, las zonas de pesca, etc., ya que a cada persona solo le tocaría una palmera con un terreno de cuatro pasos de largo por tres pasos de ancho. Con este trocito, ¿cómo podrían cultivar?, ¿y cazar?; esto de la caza sería lo más problemático, pues los animales corren de un lugar a otro y en un abrir y cerrar de ojos ya estarían en el terreno del vecino.

Era necesario encontrar otra solución. Así, una noche de luna llena se reunieron las 53 personas, que ahora vivían en la isla, con el fin de hallar una solución. Al principio todo fueron gritos y amenazas, incluso alguien fue un poco violento. Fue de nuevo Quim quien sugirió que no quedaba otra solución que ponerse de acuerdo para cazar, cultivar y todo lo que hacía referencia a la vida de todos juntos. Era necesario hacer unas normas para convivir pacíficamente y en igualdad de condiciones. Los acuerdos más básicos a los que llegaron los escribieron, e hicieron un libro que es lo que nosotros llamamos Constitución. En este libro lo primero que incluyeron fueron las cuestiones básicas en las que todos estaban de acuerdo, eran los principios de su vida en común: «En nuestra isla deseamos establecer la justicia, la libertad y la seguridad. También queremos promover el bien de todas las personas que viven aquí. Todos tenemos la voluntad de garantizar una convivencia democrática, es decir, donde todo el mundo pueda participar en las decisiones y en la organización de las cosas comunes de una manera justa».

(Isabel Carrillo \& Miguel A. Sánchez. 1992).

- Comenta con tus compañeros y compañeras los siguientes aspectos que hacen referencia a la historia que habéis leido:

1) ¿Por qué Quim quería vivir solo en la isla y consideraba intrusos al hombre y la mujer que llegaron?

2) ¿Quim tuvo que renunciar a algunas cosas y compartir otras por el hecho de vivir con otras personas?

3) ¿Crees que era importante que entre todos acordaran los principios básicos de su vida en común? ¿Por qué?

4) Con tus compañeros y compañeras de clase dialoga sobre el significado de los principios básicos que establecieron en la isla: justicia, libertad, seguridad, bienestar. 
b) Ejercicio de clarificación de valores: Frases inacabadas

- Si ya has comentado con tus compañeros y compañeras de clase el significado de los principios básicos de la vida en común - justicia, libertad, seguridad, bienestar-, ahora expresa, de forma escrita, tu opinión personal.

1. Establecer la justicia quiere decir

2. Decimos que no hay justicia cuando

3. La libertad significa.

4. Las personas no tienen libertad cuando

5. La seguridad de las personas significa que

6. La falta de seguridad es por causa de

7. El bien de todas las personas, es decir, el bienestar quiere decir

8. Cuando no todas las personas disfrutan del bienestar es por

\section{Otras actividades}

«Lo que yo haría» (Ambito temático: La diferencia, valor y conflicto. Curso: 3. ${ }^{\circ}$ de EGB)

Lee las siguientes frases y complétalas pensando lo que harías si te encontrases. ante un caso parecido.

1. Cuando veo un grupo de personas que van en silla de ruedas

2. Si un niño ciego quisiera venir a mi escuela

3. Los colegios para niños que van en silla de ruedas son

4. Si mi profesor caminase con muletas...

5. Si yo fuera presidente haría que la gente que no puede caminar

6 . Cuando juego a relevos y mi equipo pierde porque hay un niño que es muy lento, yo

7. Si no pudiera caminar, yo.

«Con mi tiempo» (Ambito temático: Escuela y trabajo. Curso: $8^{\circ}{ }^{\circ}$ )

A continuación encotrarás una serie de frases sobre el trabajo escolar, el aprovechamiento del tiempo... Piensa en lo que sueles hacer y completa las frases según tu situación.

1. Normalmente en las horas de clase yo

2. Lo que más me cuesta de la tarea escolar es porque

3. Pero, en cambio, tengo mucha facilidad para porque

4. Cuando salgo del colegio, normalmente yo....

5. Aparte de la escuela también hago

6. Los fines de semana los dedico a.....

7. Durante la semana, la hora en que suele hacer los deberes es

8. ¿Y durante el fin de semana?.

9. ¿Alguna vez no te ha dado tiempo de acabar la tarea? En caso afirmativo, explica porqué.

10. ¿Te cuesta ponerte a hacer los deberes de clase? ¿Por qué?

11. Después de haber realizado este ejercicio, ¿crees que conoces mejor el modo como te organizas el tiempo? Justifica la respuesta.

\section{Orientaciones generales para la elaboración y aplicación de ejercicios de frases inacabadas y preguntas clarificadoras}

Para la realización de estos ejercicios el educador/a, en primer lugar, ha de determinar un tema que plantee conflictos de valor y que resulte de interés para los alumnos/as. Una vez determinado el tema debe realizar un trabajo previo mediante el cual el alumno pueda adquirir información y comprender, crítica- 
mente, el conflicto que se plantea así como las diversas posiciones respecto a él. Por último ha de motivar el proceso de valoración personal a partir de ejercicios de clarificación de valores. Para ello ha de establecer un clima psicológicamente seguro, es decir, ha de crear un ambiente de confianza y respeto, donde se acepte y se fomente la diversidad de opiniones y donde no se obligue a nadie a expresar lo que piensa y siente. También habrá de motivar a los alumnos/as para que sean sinceros en su proceso de valoración, en sus respuestas. Esta motivación puede lograrse atendiendo a dos aspectos: a) Que los temas de reflexión se vinculen a los problemas que vivencian los alumnos/as o se refieran a aquellos otros temas por los que están altamente interesados; b) Que los alumnos/as perciban los beneficios que comporta la realización de estos ejercicos. En este sentido se les puede indicar que es un medio que permite conocerse mejor y, a partir de aquí, establecer pautas de cambio que posibilitan mejorarnos a nosotros mismos y, con ello, mejorar las relaciones con los demás.

Por lo que se refiere a la elaboración de las actividades, únicamente indicar que cada ejercicio se realizará a propósito de un tema concreto (no es conveniente elaborar una actividad que aborde diversos temas, de forma simultánea, ya que ello puede crear cierta confusión y dificultar el proceso de valoración). Las frases inacabadas y/o las preguntas clarificadoras han de redactarse de tal forma que no induzcan a dar una respuesta determinada; tampoco han de ser ambiguas ya que con ello podemos provocar cierta desorientación. Las frases y preguntas han de redactarse de forma concreta y sencilla, requiriendo por parte del alumno/a una respuesta breve y clara que ponga de manifiesto lo que valora y aprecia. Su extensión - número de frases y preguntas - no ha de ser excesiva. Alrededor de diez frases o preguntas - dependiendo de la edad de los alumnos/as-, por actividad, resulta adecuado y suficiente para reflexionar acerca de las diferentes dimensiones que puede presentar un tema determinado.

\section{Notas}

${ }^{1}$ En 1967 se publica en lengua castellana la obra de L. Raths, M. Harmin y S. B. Simon con título «El sentido de los valores y la enseñanza».

${ }^{2}$ El conocimiento de sí mismo, y la toma de conciencia respecto a lo que cada uno valora y aprecia, puede ser el primer paso para establecer una relación coherente entre juicio y acción moral.

${ }^{3}$ Junto a las frases inacabadas y preguntas clarificadoras, otros dos métodos permiten poner en juego el proceso de valoración personal: a) Diálogos clarificadores: Son ejercicios orientados a profundizar en las reflexiones que realiza el alumno/a. El educador/a, mediante preguntas y comentarios, ha de conseguir que el alumno/a establezca un diálogo consigo mismo para que, con ello, examine su conducta, sus ideas y decida lo que él desea. b) Hoja de valores: Consiste en presentar un breve texto en el que se expone una situación problemática, con una serie de preguntas, que invita a la discusión. El objetivo es dialogar acerca del problema planteado exponiendo lo que cada uno valora respecto a él.

${ }^{4}$ Este podría ser el trabajo previo a lo clarificación de valores al que nos referíamos anteriormente. En esta actividad, mediante una historia ficticia, se ofrece información referida a cómo nace una Constitución y cuáles son los principios básicos que rigen la vida en común. 


\section{Referencias}

Carrillo, I.; Galceran, M. del M.; Lopez, S.; Martín, X.; Paya, M.; Puig, J. M. a ; Vilar, J. (1992). «Los contenidos». CUADERNOS DE PEDAGOGIA, n.0 201, marzo 1992. págs. 15-18.

Curwin, R. L.; Curwin, G. (1984). Cómo fomentar los valores individuales. Barcelona. CEAC.

Hersh, R.; Reimer, J.; PAoltro, D (1988). El crecimiento moral. De Piaget a Koblberg. Madrid. Narcea.

Pascual, A. V. (1988). Clarificación de Valores y Desarrollo Humano. Madrid: Narcea.

Puig, J. M. ${ }^{a}$; Martinez, M. (1989). Educación moral y democracia. Barcelona: Laertes.

Raths, L.; Harmin, M.; Simon, S. B. (1967). El sentido de los valores y la enseñanza. México. Uthea.

VILAR, J. «Clarificación de valores» en MARTINEZ, M.; PUIG, M. M. ${ }^{a}$ (coord.) (1991). La educación moral. Perspectivas de futuro y técnicas de trabajo. Barcelona: Graó. ICE. UB. págs. 83-43.

\section{Análisis crítico del entorno, elección de alternativas y clarificación de valores Isabel Carrillo Flores \\ CL\&E, 1992, 15, pp. 77-83}

Resumen: La técnica de clarificación de valores, cuyo orígen se situa en las aportaciones de L. Raths y sus colaboradores (1966), engloba un conjunto de métodos cuya finalidad es ayudar a los alumnos/as a realizar un proceso de reflexión -interno y externo- que les permitirá tomar conciencia de los propios pensamientos, valores, sentimientos... Este proceso es necesario para que la persona, de forma autónoma y racional, se descubra a sí misma y vaya construyendo su personalidad moral.

Datos sobre el autor: Isabel Carrillo Flores es pedagoga y actualmente goza de una beca de Formación Personal Investigador de la Generalitat de Catalunya. Tambien es miembro del Grupo de Investigación en Educación Moral (G.R.E.M.).

Dirección: Departamento de Teoría e Historia de la Educación, Facultad de Pedagogía, Universidad de Barcelona, Baldiri Reixac, s/n 08028 Barcelona. Tel (93) 3333466.

(C) De todos los artículos deberá solicitarse por escrito autorización de CL\&E y de los autores para el uso en forma facsímil, fotocopia o cualquier otro medio de reproducción impresa. CL\&E se reserva el derecho de interponer las acciones legales necesarias en aquellos casos en que se contravenga la ley de derechos de autor. 